This paper has been published in The International Journal of Advanced Manufacturing Technology, August 2014.

DOI: 10.1007/s00170-014-6248-y

Borhen Louhichi, Gad N. Abenhaim, Antoine S. Tahan.

\title{
CAD/CAE integration: Updating the CAD Model after a FEM analysis
}

\author{
Borhen Louhichi, Gad N. Abenhaim, Antoine S. Tahan \\ LIPPS, ÉTS, 1100, Notre-Dame Ouest, Montréal, H3C1K3, Québec, Canada \\ borhen.louhichi@etsmtl.ca, 1borhen@gmail.com, antoine.tahan@etsmtl.ca
}

\begin{abstract}
The improvement of the simulation process requires an integration of the design and analysis models. There are two essential tasks in the design analysis process: (i) Computer Aided Design (CAD) which provides the geometric description of the model and (ii) the Finite Element Method (FEM) used for mechanical behaviour simulations. The interoperability between these two tasks reduces costs and improves product quality through the acceleration of design analysis loops. Our activity fits into this research orientation by providing a method to link the FE analysis and the CAD model. This is done by reconstructing the CAD model from the FE analysis results (deformed mesh). This paper proposes a method to update the CAD geometry from the deformed mesh. This approach allows for rebuilding the CAD model after analysis by extracting geometric information from the deformed mesh. An illustration of the developed method is discussed at the end of this paper.
\end{abstract}

Keywords: CAD model, mesh, WDE algorithm, B-Spline surface, reconstruction, assembly.

\section{Introduction}

The complete definition of a product often requires the collaboration and the integration of various tools/activities (CAD, CAM, analysis...). Data sharing and exchange between tools/activities has thus become an important task throughout a product's life cycle. The CAD model is one of the most important models used throughout the product's entire life cycle. It represents the geometric support used in many other activities (analysis, manufacturing, assembly, etc.). In particular, mechanical analysis is one of the most important activities associated with design that reduces product development times and production costs.

In CAD software (CATIA, Solidworks...), the outcomes from CAE (analysis) to CAD are ISO values, deformed mesh... etc. However, these results are just a discrete representation and are not the most appropriate for future use. Indeed, we need quantitative results to use in CAD/CAM environment and operating assembly simulations. Thus, a more generic description has to be provided and the CAD description is the most relevant choice because of its underlying formalism. It is therefore the designer's responsibility to take them into account in the design and validation of CAD models. 
This research aims to improve $\mathrm{CAD} / \mathrm{CAE}$ integration by reconstructing a CAD model from $\mathrm{FE}$ analysis results (i.e. deformed mesh). This reconstruction is necessary to obtain a geometric model as a mathematical model from the mesh information (i.e. data points, triangulations etc). This model is used to verify and validate the part's design in a deformed state. It makes it possible to visualise and simulate the behaviour of mechanical assemblies in their deformed configuration (normal operating state) and to detect possible interference and collision effects between parts which are undetectable in the non-deformed state [1].

This paper is organized as follows. First, a literature review covering the CAD/CAE integration and the reconstruction of the CAD model entities (surfaces/faces, curves/edges) in particular is presented. Next, the proposed approach and algorithms utilized to rebuild the geometry of the CAD model are detailed, followed by the model validation results. Conclusions and perspectives are presented at the end.

\section{State of the art}

This paper can be positioned with respect to research done in two areas: The CAD/CAE integration and the reconstruction of geometric entities, in particular, the surface reconstruction necessary to rebuild the CAD model.

\subsection{CAD/CAE integration}

Several research investigations have been carried out to improve CAD/CAE integration: (i) simplification of a CAD model through the elimination of its small details in order to perform a FE analysis [2], (ii) generation of a Finite Element Mesh from Stereolithography (STL) files [3], and (iii) the iso-geometric analysis method [4]. The latter approach deals with generating a deformed solid model, which is the focus of this paper. The principle of this approach is to use the characteristics of B-Spline as a support for the deformation analysis, thus removing the need for creating a mesh from a CAD model. The deformed model resulting from this analysis is a CAD model on its own. Therefore, the need to reconstruct a CAD model from FEM results, which is encountered with FEM-based approaches, is avoided in the iso-geometric analysis method. Although this approach avoids the need to generate specific mesh, it is restricted to the two-dimensional problems of linear elasticity and solids with a simple topology. In the same context (i.e. iso-geometric analysis), Rypl and Payzak [5] introduce a new methodology for the construction of a weighted dual graph of a two-dimensional NURBS-based isogeometric mesh. The similarity between the IGA (isogeometric analysis) and FEA allows the IGA to adopt the same parallelization concepts and paradigms as the FEA. Therefore this new methodology enables the use of standard graph-based partitioning approaches to obtain a balanced decomposition with minimized communication costs which can be adopted for parallel isogeometric analysis.

\subsection{Surface reconstruction}

Various works have investigated the problem of surface deformation. These include the work by Bloor [6] which introduces deformable B-Splines and the D-NURBS (Dynamic NURBS) developed by Qin [7]. In 2004, LaGreca [8] dealt with the deformed B-Spline problem in his research on geometric modelling. Song et al. [9] propose a technique for modeling implicitly defined surfaces with sharp features. They suppose that an initial surface is generated from an unorganized point cloud and then sharp edges and vertices in the initial surface are detected. Then the edges and vertices descriptors are added. In the end, sharp features are represented after an evolution process is used for fitting the edge curves. Xiea et al. [10] propose a new approach, a 
combination of a hybrid optimization algorithm and an iterative scheme (HOAAI) for the reconstruction of 3D surfaces from 3D data points based on non uniform rational B-Splines. This approach is based on an algorithm for optimizing the weights and parameterization of the data points, an iterative scheme to compute the knot vectors and a method for Boundary-determined parameterization.

This literature review relates to two different aspects. The first aspect consists of CAD/CAE integration [1-5]. The second aspect consists of the CAD entities reconstruction, in particular the surface reconstruction which is the most delicate task in the CAD model reconstruction [6-10]. In most of the references cited, the $\mathrm{CAD} / \mathrm{CAE}$ integration field does not lead to the reconstruction of a geometric model as an analysis result. The iso-geometric analysis method seems interesting but it is restricted to two-dimensional models. In the cited references dealing with the second aspect (i.e. surface reconstruction), the work of Xiea et al. [10] seems interesting in regards to surface reconstruction from an unorganized point cloud, especially if we don't have an initial surface to update.

In this work, we focus on updating the nominal CAD model from the FE analysis results (deformed mesh). In this context, the topology of the CAD model does not change. That is to say, the number of faces (resp. edges) of the reconstructed deformed CAD model is the same as the number of faces (resp. edges) of the un-deformed CAD model; Only the geometry changes and should be updated to obtain the deformed CAD model. Taking advantage of this characteristic, this paper proposes a method that updates the nominal CAD model using a Boundary REPresentation (BREP model) of the nominal model and the displacements values of the FE model nodes retrieved from the FE analysis results. The proposed method updates the nominal BREP model control points' positions by the values obtained from the weighted displacement estimation method

\section{Reconstruction of the CAD model from FEM results}

\subsection{General algorithm reconstruction}

The general algorithm allows for the reconstruction of CAD model as a Boundary REPresentation (BREP model). The BREP model describes not only geometrical information (surfaces, curves and points), but also topological information (faces, edges and vertices). The proposed algorithm is based on two main parts: to determine the topology and rebuild the geometry. We first build the BREP entities and then join them to build the deformed CAD model. BREP entities are defined using deformed mesh boundary nodes (faces mesh). 


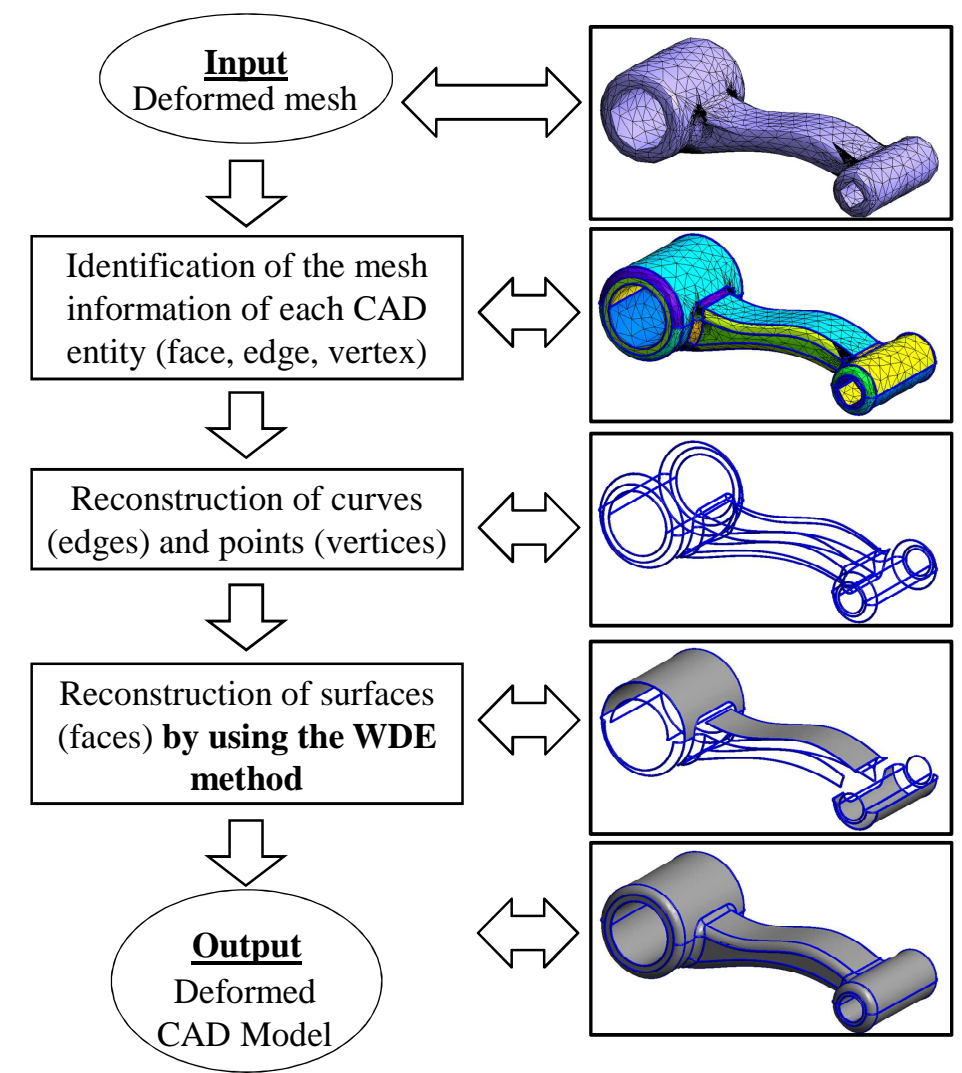

Fig. 1: Reconstruction algorithm

The general algorithm (Fig. 1) of reconstruction exploits information extracted from a triangulated surface obtained from the deformed mesh. In addition, a topological model describing how surfaces are inter-connected is provided. The main steps of the reconstruction algorithm are as follows:

- Identification of the mesh information for each CAD entity;

- Reconstruction of the CAD edges and vertices;

- Reconstruction of the CAD faces.

The last step is the most complicated phase in the CAD model reconstruction. Indeed, there are many difficulties in using a direct interpolation method to generate the CAD face (surface) from the mesh: (i) the data modeling the surface consists of a triangulation of an unorganized set of points in the parametric space $(\mathrm{u}, \mathrm{v})$ of the reconstructed surface; (ii) the density of information (triangles, points) is not constant over the surface; (iii) in the case of faces with inner loops it is not easy to evaluate the surface, and mesh information (nodes) is not sufficient to calculate BSpline shape functions. Thus the WDE method is used to solve any encountered difficulties and update the CAD surfaces (faces) after deformation.

\subsection{CAD edge reconstruction}

Reconstruction of the CAD edges is obtained by an interpolation of points extracted from the boundary line of a triangulated surface. We first start by giving details about the interpolation method used in this paper. Because CAD surfaces commonly encountered are based on B-Spline 
surfaces, the edges we will generate must be compatible with their faces so reconstructed surfaces must be smooth on the curves. B-Spline curves are used to interpolate the point to obtain edges.

\section{$B$-Spline curve interpolation}

Suppose we have $n$ points $Q_{k}$ to interpolate with a $p^{\text {th }}$ degree B-Spline curve. Each interpolation point is described by the equation:

$$
Q_{k}=C\left(\overline{\mathrm{u}}_{k}\right)=\sum_{i=0}^{n} N_{i p}\left(\overline{\mathrm{u}}_{k}\right) P_{i}
$$

Where $P_{i}$ are the control points of the curve, $N_{i}$ are the shape functions and $\bar{u}_{k}$ is the parameter of the $Q_{k}$ on the curve $\left(0 \leq \bar{u}_{k} \leq 1\right)$. In order to solve this equation $E q$. 1, we can choose the $\bar{u}_{k}$ of each $Q_{k}$ on the curve and calculate an appropriate knot vector. There are three methods for choosing the $\bar{u}_{k}$ parameters [11] [12]: the equally spaced method, the chord length method and the centripetal method (Fig. 2). To reconstruct the CAD edges, the centripetal method is used. It provides the best parameterization and has the most stable behaviour when the data takes very sharp turns [11] [12]. This method is described by the following equation:

Let $d$ be the total of square of length:

$$
d=\sum_{k=1}^{n} \sqrt{\left|Q_{k}-Q_{k-1}\right|}
$$

Then

$$
\overline{\mathrm{u}}_{0}=0, \overline{\mathrm{u}}_{n}=1, \overline{\mathrm{u}}_{k}=\overline{\mathrm{u}}_{k-1}+\frac{\sqrt{\left|Q_{k}-Q_{k-1}\right|}}{d}, \quad(k=1 \ldots n-1)
$$

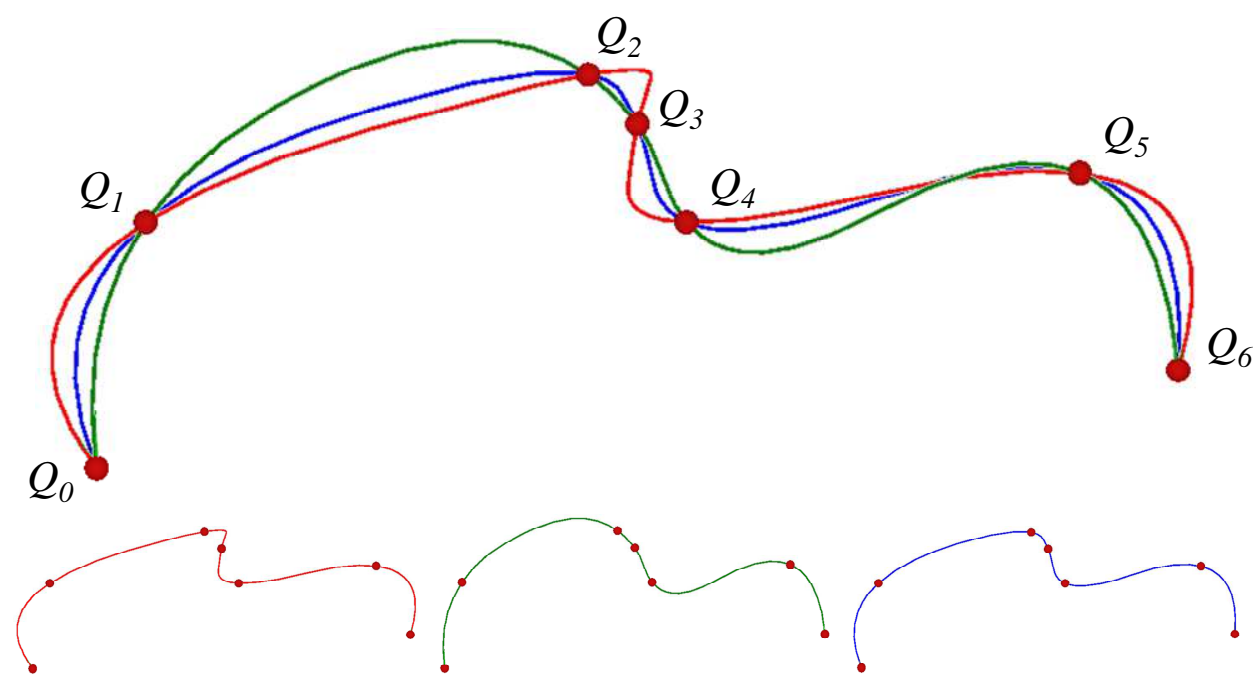

(a)

(b)

(c)

Fig. 2: Curve interpolation with different parameterization methods: (a) equally spaced, (b) chord length, (c) centripetal [12].

In the bibliography [11] [12], the averaging method is used to compute the knots vector from the $\bar{u}_{k}$ parameters. By using this method, knots reflect the distribution of the data points, which is important in order to gain accuracy in the interpolation:

$$
\left(u_{0}=\ldots=u_{p}=0, u_{m-p}=\ldots=u_{m}=1\right), \quad u_{j+p}=\frac{1}{p} \sum_{i=j}^{j+p-1} \overline{\mathrm{u}}_{i}, \quad(j=1 \ldots n-p)
$$


Knot vectors are used to compute the B-Spline shape functions. In the last step, the control points of the curve are computed by solving Eq. 4 .

\subsection{CAD faces reconstruction}

The faces reconstruction method is based on the B-Spline surface and all the model faces are approximated by NURBS because it's the most appropriate surface that defines the deformed faces. In fact the CAD face (surface) in the CAD model must move and deform, when the mesh in the F.E model deforms. So, it's easy to deform a B-Spline surface and it's not possible in the case of the primitive faces (plane, cylinder...). In additional, the most CAD systems use B-Spline surfaces to model the faces of the CAD model. So, the reconstruction of the CAD faces is based on the B-Spline surface. We will now describe the CAD face reconstruction algorithm. It is based on the following steps (Fig. 3): 


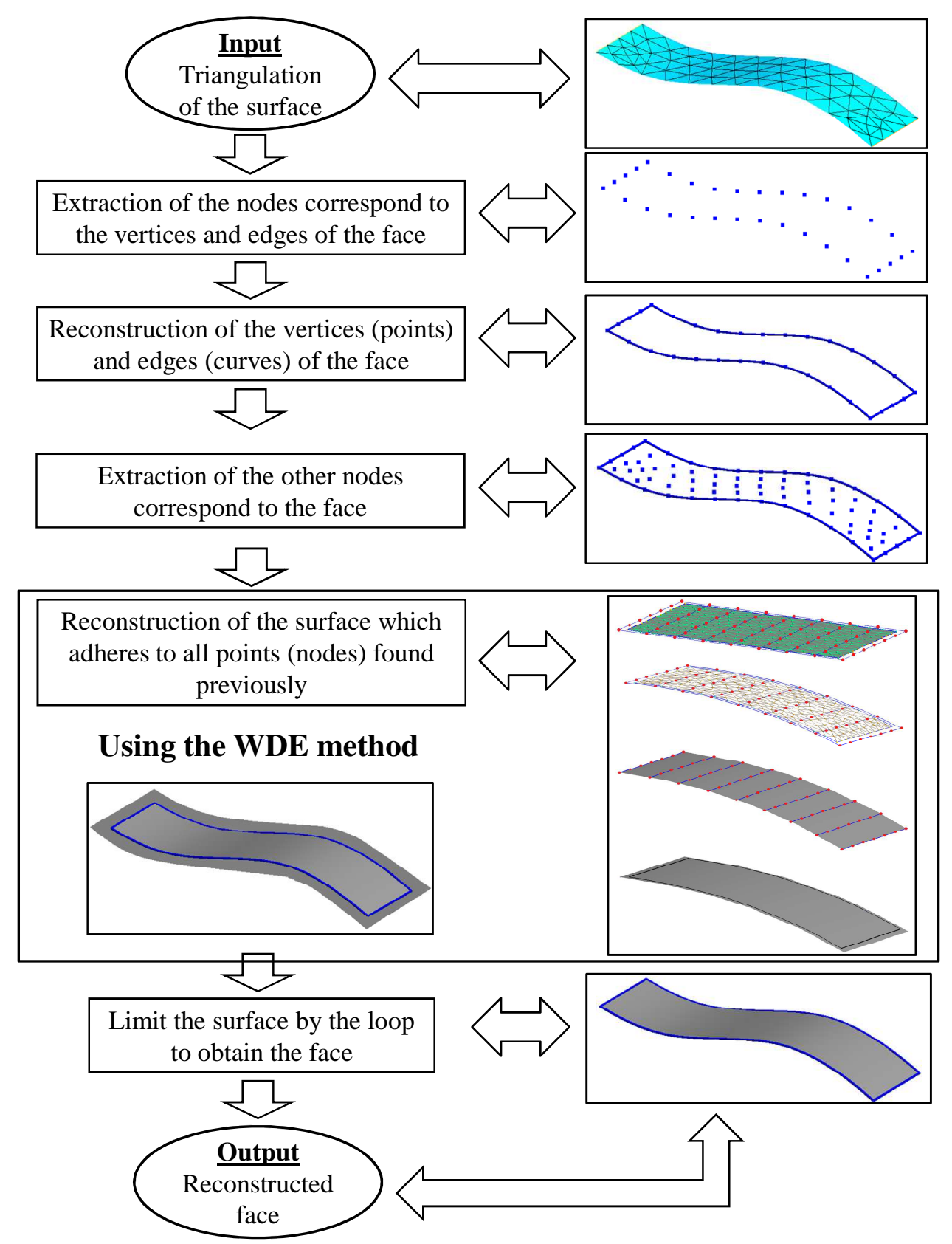

Fig. 3: Reconstruction algorithm of the CAD model faces

- Identification of the triangulation associated with each face of the model;

- Extraction of the nodes corresponds to the vertices and edges of the face;

- Reconstruction of the vertices and edges of the face;

- Extraction of the nodes correspond to the face;

- Reconstruction of the deformed surface;

- Generation of the 2D edges corresponding to face loops by projection of the 3D edges previously computed;

- Trim the reconstructed surface by the loop to obtain the face. 
To use the B-Spline surface type for modeling the faces, we need a regular lattice of control points. It is necessary to compute the two knot vectors in the parametric space $(\mathrm{u}, \mathrm{v})$ and to find the shape functions. The biggest difficulty encountered is that the data modeling of the surface consists of a triangulation of unorganized set points in the parametric space $(\mathrm{u}, \mathrm{v})$ of the reconstructed surface.

Consequently, a direct interpolation method cannot be used. We must compute a regular lattice from the triangulation points (data points) from which the B-Spline parameters will be computed. Solving these problems consists of defining a regular lattice of control or interpolation points that replace the triangulation to define the B-Spline surface. Thus the key idea is to generate the regular lattice of points on the initial surface (face before deformation). Thereafter, these points must be moved and the moved lattice is used to compute the B-Spline surface of the deformed face. The question is: How do we move points of the lattice to compute the coordinates of structured interpolation points of the B-Spline surface through smoothed triangulation? To address this challenge, this paper proposes a weighted displacement estimation (WDE) method.

\subsection{The weighted displacement estimation (WDE) method}

The weighted displacement estimation method updates the position of the B-Spline surface lattice control points $\left(P^{\circ}\right)$ by estimating their displacement $(\delta)$ using the weighted mean displacement induced to neighboring nodes of the mesh model during the FE analysis. By noting the coordinates of the nodes of the meshed CAD model as $M^{\circ}$ and their coordinates after a FE analysis as $M^{\bullet}$, the weighted displacement estimation of each control point $\left(P_{j}^{\circ}\right)$ associated with a surface $\left(S^{k}\right)$ is formulated as follows;

$$
\delta_{j}=\frac{\sum_{i}^{n_{j}}\left\|P_{j}^{\circ}-M_{j i}^{\circ}\right\|\left\{M_{j i}^{\circ}-M_{j i}^{\circ}\right\}}{\sum_{i}^{n_{j}}\left\|P_{j}^{\circ}-M_{j i}^{\circ}\right\|} \quad \forall j \mid P_{j}^{\circ} \in P^{\circ}
$$

Where $M_{j}^{\circ}$ is the set of $n_{j}$ neighboring nodes encompassed within a radius $R_{j}$ of the control point $P_{j}^{\circ}$ :

$$
\left\|P_{j}^{\circ}-M_{j i}^{\circ}\right\| \leq R_{j} \quad \forall i \mid M_{j i}^{\circ} \in M^{\circ}
$$

Such as the nodes $M_{j}^{\circ}$ and the control points $P_{j}^{\circ}$ are associated to the same surface $\left(S^{k}\right)$. The radius $R_{j}$ is set depending on the surface curvature and the lattice grid.

Having estimated the displacement $\delta$, the updated B-Spline surface lattice control points $\left(P^{\bullet}\right)$ representing the deformed $\mathrm{CAD}$ model (i.e. NURBS surface fitting the output-mesh of the FEA) is evaluated by;

$$
P_{j}^{\bullet}=P_{j}^{\circ}+\delta_{j} \quad \forall j \mid P_{j}^{\circ} \in P^{\circ}
$$


The phase to update the CAD face after deformation is based on the following steps:

- Generating a regular lattice (in the $u, v$ parametric space) of points on the initial CAD face (before deformation);

- Moving the regular lattice of points by using the WDE method to fit the deformed mesh corresponding to face (Fig. 4);

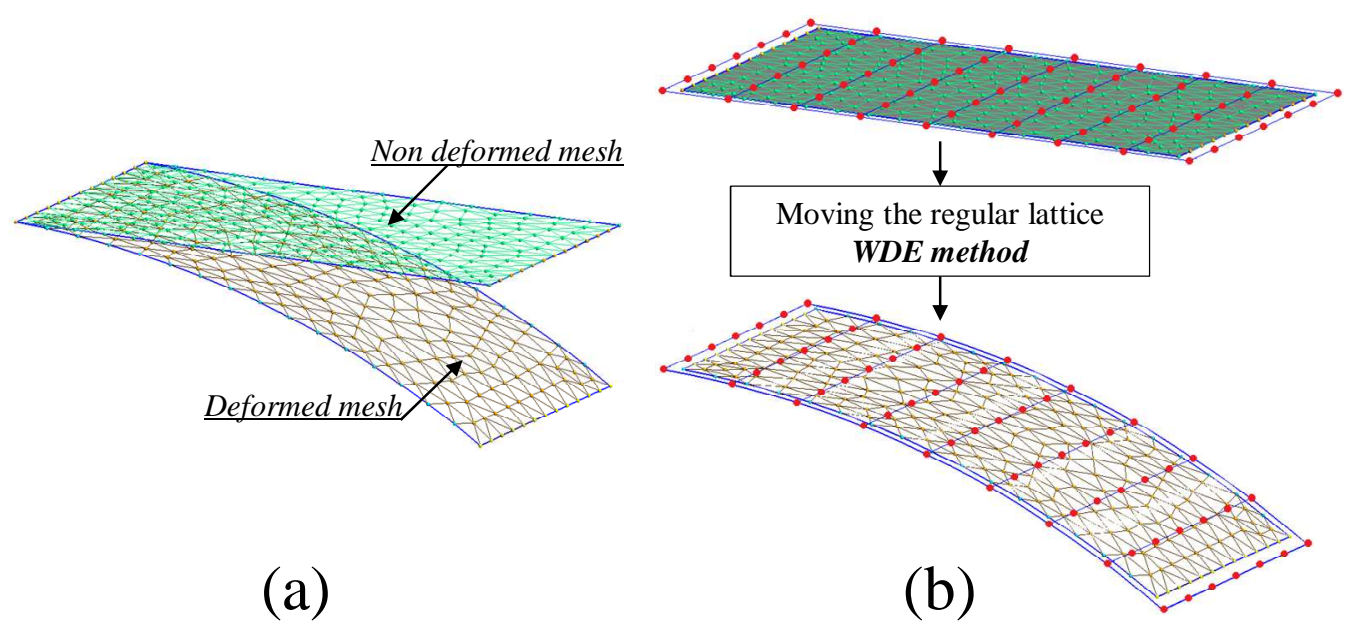

Fig. 4: (a) initial mesh and deformed mesh, (b) Moving the regular lattice by using the WDE method.

- Computation of the parameters of the B-Spline surface and reconstruction of the deformed CAD face (Fig. 5): (i) generation of the 2D edges corresponding to face loops, (ii) trim the reconstructed surface by the loop: If the loop is an outer loop of the face, the part of the surface outside the loop is not considered in the face. Also, if the loop is an inner loop, the part of the surface inside the loop is not considered.

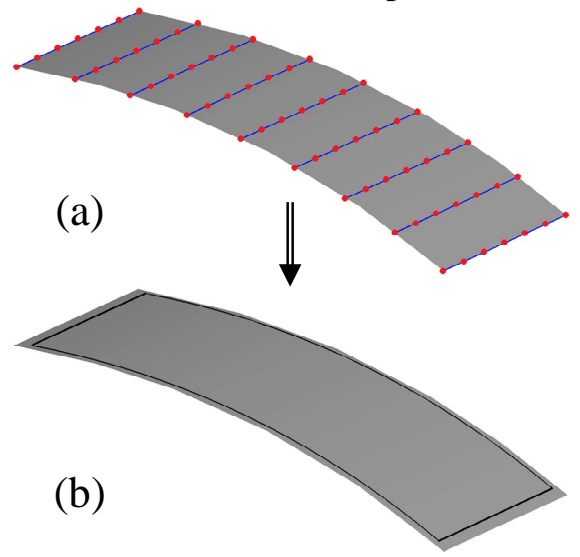

Fig. 5: (a) Reconstruction of the deformed surface (b) Adding the loop to obtain the deformed face.

The regular lattice of the interpolation points computed in the previous step is used to compute the parameters (parameters $\left(\bar{u}_{k}, \bar{v}_{l}\right)$ of each points, knots vectors and control points) of the BSpline surface.

We are given a set of $(n+1)(m+1)$ data points $Q_{k l}^{\text {lattice }}, k=0 \ldots n$ and $l=0 \ldots m$, we want to construct a $(p, q)^{\text {th }}$ degree B-Spline surface interpolating these points [12].

In the first step, parameters $(u, v)$ of the data points are calculated by using the centripetal method Eq. 9. In the second step, an averaging method is used to compute the knots vectors Eq. 10 [12]. 
These methods used to compute the parameters and the knots vectors guarantees the best results [12].

To compute the coordinates of the control points $P_{i j}$ the following equation is used [12]:

$$
\begin{aligned}
Q_{k l}^{\text {lattice }}=S\left(\bar{u}_{k}, \bar{v}_{l}\right) & =\sum_{i=0}^{n} N_{i p}\left(\bar{u}_{k}\right)\left(\sum_{j=0}^{m} N_{j q}\left(\bar{v}_{l}\right) \cdot P_{i j}\right) \\
Q_{k l}^{\text {lattice }} & =S\left(\bar{u}_{k}, \bar{v}_{l}\right)=\sum_{i=0}^{n} N_{i p}\left(\bar{u}_{k}\right) R_{i l} \\
R_{i l} & =\sum_{j=0}^{m} N_{j q}\left(\bar{v}_{l}\right) \cdot P_{i j}
\end{aligned}
$$

Algorithms used to compute the B-Spline surface parameters from a regular lattice of points are described in the NURBS Book [12].

The WDE method is used to solve two problems: i) the problem of point regularization - in fact, it is not obvious to interpolate the B-Spline surface directly from the mesh nodes; ii) the problem of faces with inner loops - in fact, in this case, mesh information (nodes) is not sufficient to calculate the B-Spline shape functions that are necessary to calculate the control points of the B-Spline surface from the interpolation points.

\section{Illustration}

The proposed approach is developed by using Matlab and The Solidworks API (Application Programming Interface). The development is based on three main steps:

- Extraction of the mesh information from the FE results obtained by Solidworks Simulation and identification of the mesh information corresponding to each CAD entity (face, edge, vertex). This step is developed by using Solidworks API;

- Computation of the CAD entities (interpolation points, control points...) by using the information extracted in the last step. This step is developed under Matlab;

- Generation of the geometry and the topology of the CAD entity to obtain the final CAD model. This step is developed by using Solidworks API.

Two case studies are used to evaluate the proposed method's performance: a rod (crankshaft assembly) and a rocker toggle (mechanical part). Figure 7. (a) and Figure 11. (a) illustrate the cases. In addition to the nominal CAD model, the un-deformed and deformed meshes used in the FE analysis are provided for each case study.

\subsection{Example1- crankshaft assembly}

The first case represents the connecting rod of a crankshaft assembly. Figure 6 (b) presents the board conditions defined on the connecting rod CAD model. The connecting rod is loaded in compression (under prevailing gas pressure) and in tension (primarily due to inertia force). The analysis is realized in the compression phase (Fig $7 \mathrm{c}-\mathrm{d}$ ).

After the extraction of the deformed mesh, the reconstruction of the curves (edges) is realised. It is a direct interpolation from the mesh information (section 3-2). To rebuild the surfaces (faces), the WDE method is used to move a regular lattice (computed on the initial surface) representing the interpolation points of the B-Spline surface. Face reconstruction is based on three steps:

- Generation of a regular lattice (in the $u, v$ parametric space) of points on the initial CAD face before deformation (Fig. 6 (a)); 
- Moving the regular lattice of points by using the WDE method to fit the deformed mesh corresponding to face (Fig. 6 (c));

- Computation of the B-Spline surface parameters and reconstruction of the deformed CAD face (surface limited by loops - Fig. 6 (d)).
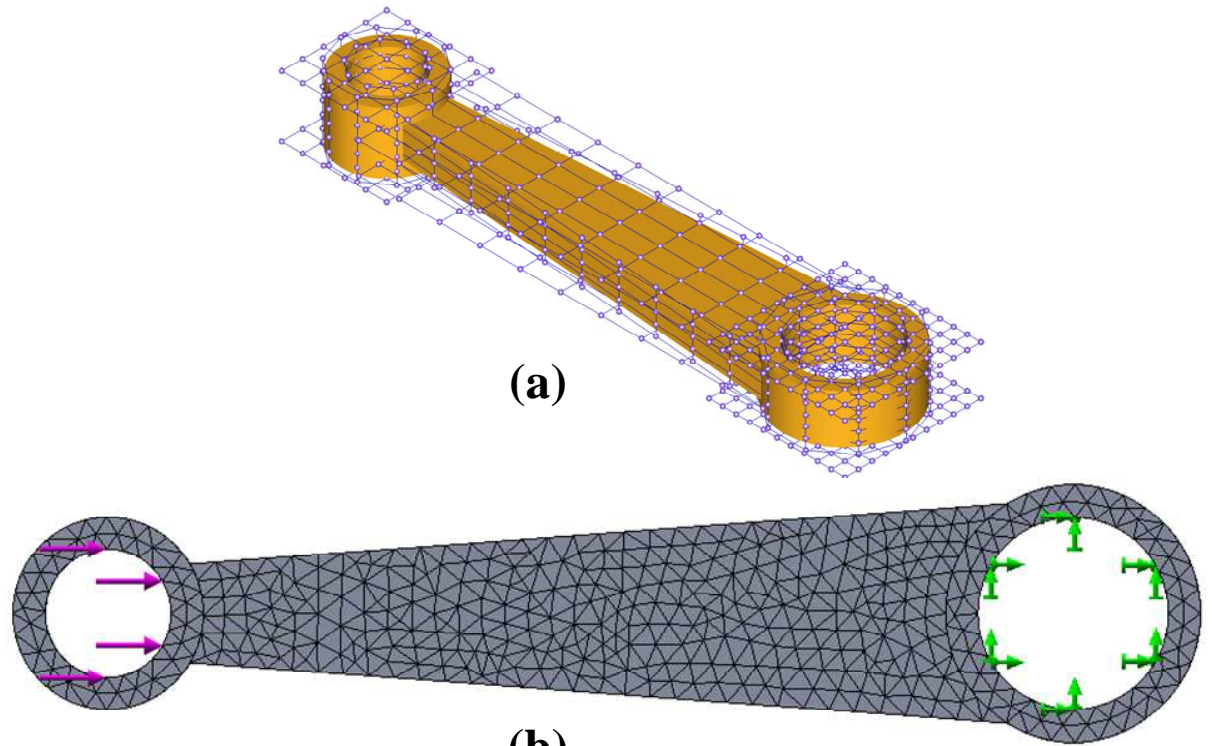

(b)

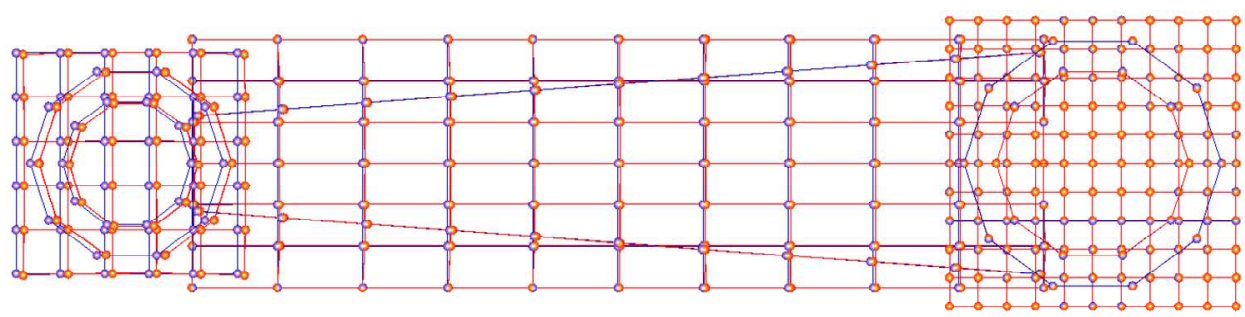

-

- $\downarrow$ Deformed lattice obtained by WDE method

(c)

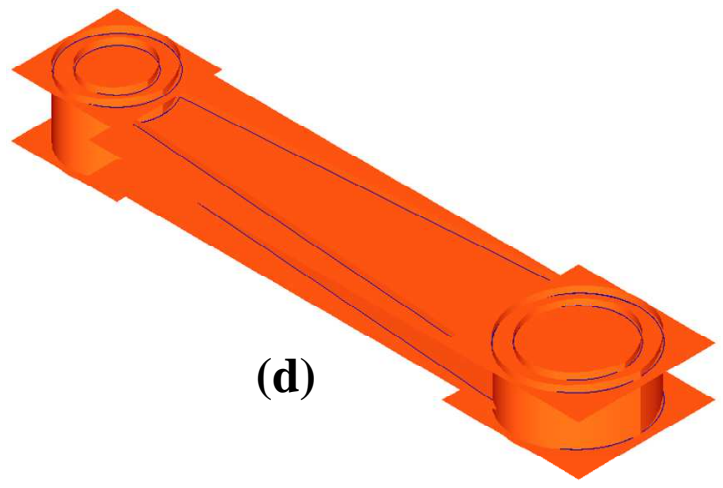

Fig. 6: Connecting rod model. 
Figure 7 (e) presents a rebuilding of the deformed CAD model of the connecting rod. New axes are computed on the deformed connecting rod model. This allows defining new mating constraints between the deformed part (connecting rod) and other parts of the assembly.

The assembly with deformed parts allows a virtual simulation of the assembly functioning (rod crankshaft) with the deformed model (realistic model in the operating conditions). According to this simulation, the designer can discern possible collisions between parts, particularly between the connecting rod and crankshaft, and between the connecting rod and piston axis (Fig 7(f)).

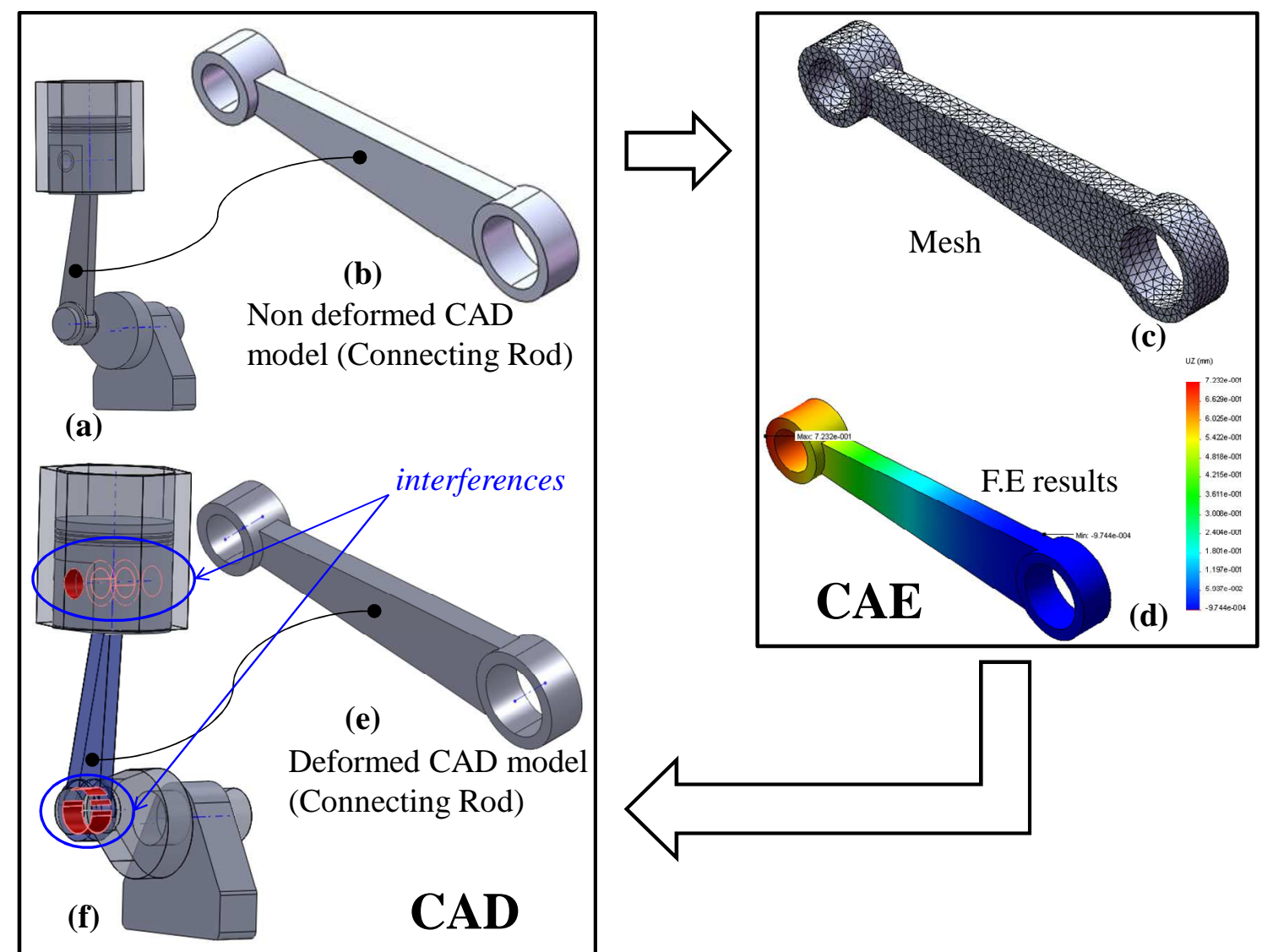

Fig. 7: Illustration method - crankshaft assembly.

Table 1, Fig. 8, Fig. 9, and Fig. 10 illustrate many quantitative criteria and results obtained by the reconstruction algorithm of the previous example (Fig. 6). The reconstruction error is the distance between the interpolation point (node) and its projection on the reconstructed surface. The calculated errors are negligible compared to the node displacements. 

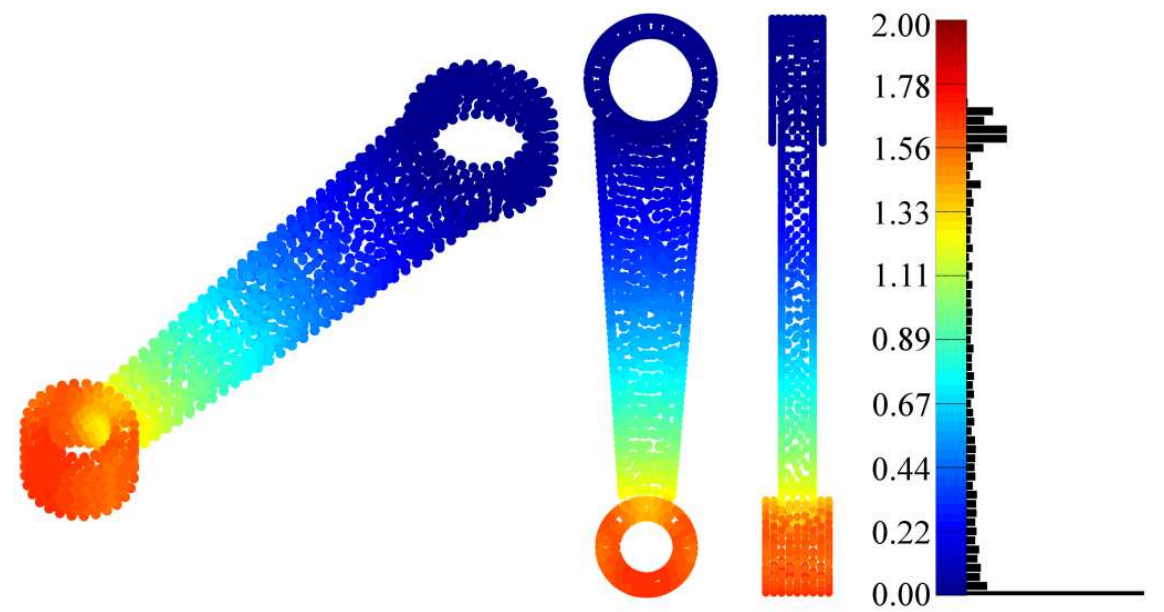

Fig. 8: Colormap and its distribution of the surface nodes FEA displacements results in $10^{-1} \mathrm{~mm}$.

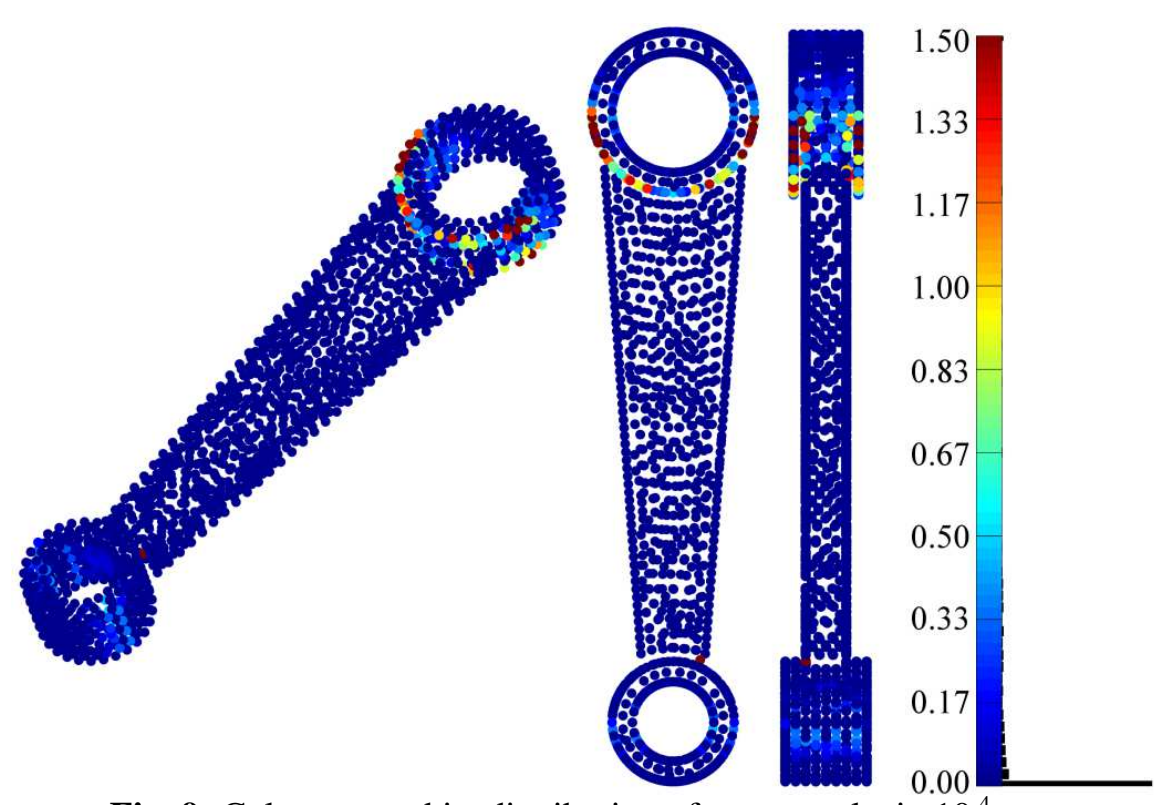

Fig. 9: Colormap and its distribution of error results in $10^{-4} \mathrm{~mm}$. 


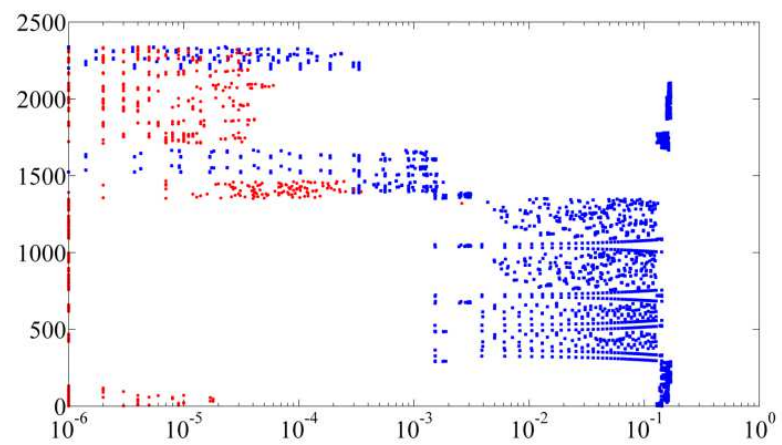

Fig. 10: Error results (Red) and FEA displacement results (Bleu) of each surface nodes in millimeters. Vertical axis is the nodes identification number; the logarithmic horizontal axis is in millimeters.

Table 1: Quantitative criteria and results (Connecting rod).

\begin{tabular}{|c|c|}
\hline Number of faces & 16 \\
\hline Node displacements (analysis result) & $0.000 \rightarrow 0.17 \mathrm{~mm}$ \\
\hline Maximal reconstruction error & $0.002 \mathrm{~mm}$ \\
\hline
\end{tabular}

\subsection{Example2- rocker toggle Part}

The second case represents the rocker toggle. It is selected as an example of validation. Figure 11 (c) presents the board conditions defined on the rocker toggle CAD model. The analysis is realized and finite elements results are extracted. Figure 11 (d) presents one F.E results.

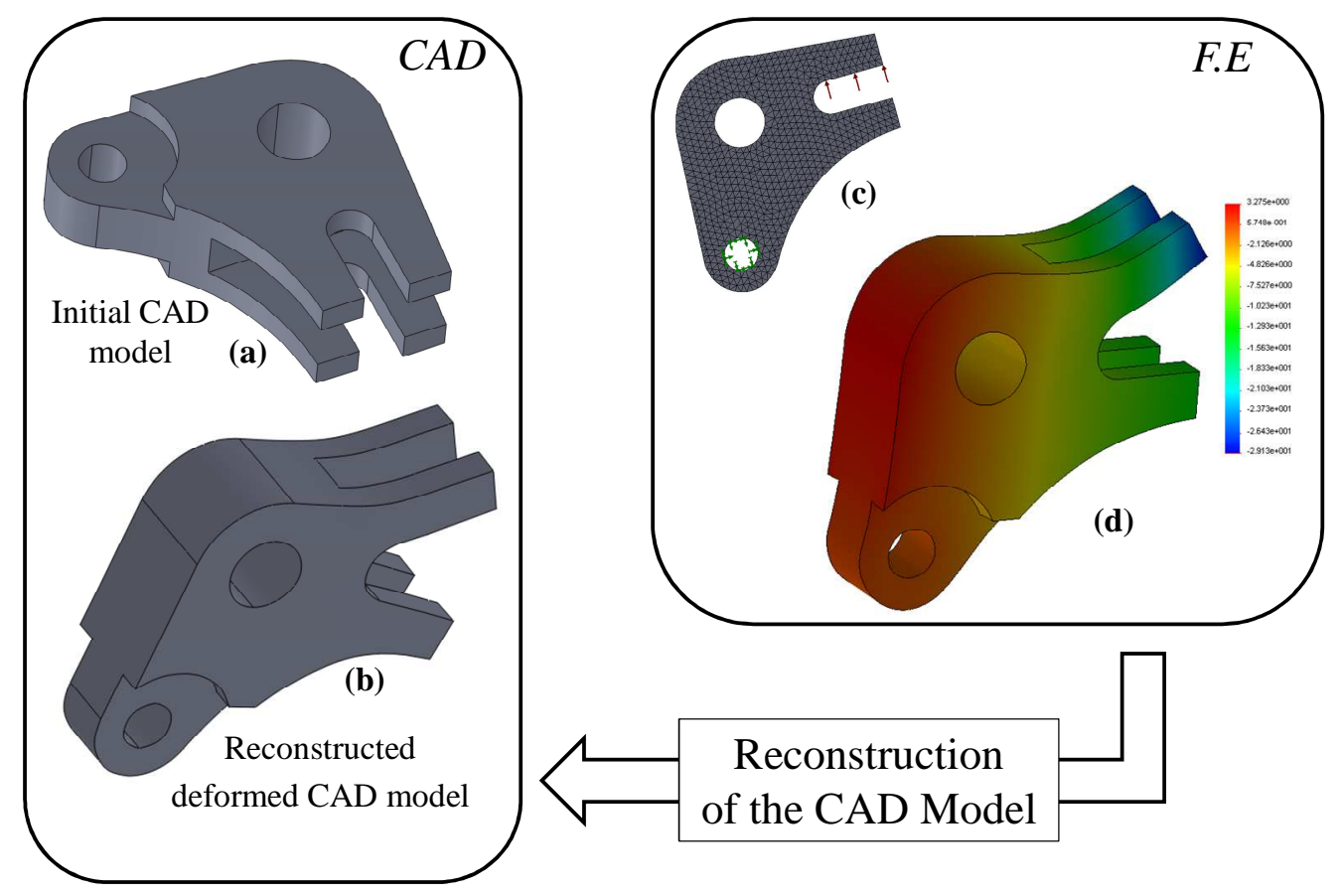

Fig. 11: Illustration method - rocker toggle. 
Figure 12 presents the face reconstruction steps:

- Generation of a regular lattice (in the $u, v$ parametric space) of points on the initial CAD face before deformation (Fig. 12 (a));

- Moving the regular lattice of points by using the WDE method to fit the deformed mesh corresponding to face (Fig. 12 (b));

- Computation of the B-Spline surface parameters and reconstruction of the deformed CAD face (surface limited by loops - Fig. 12 (c)).

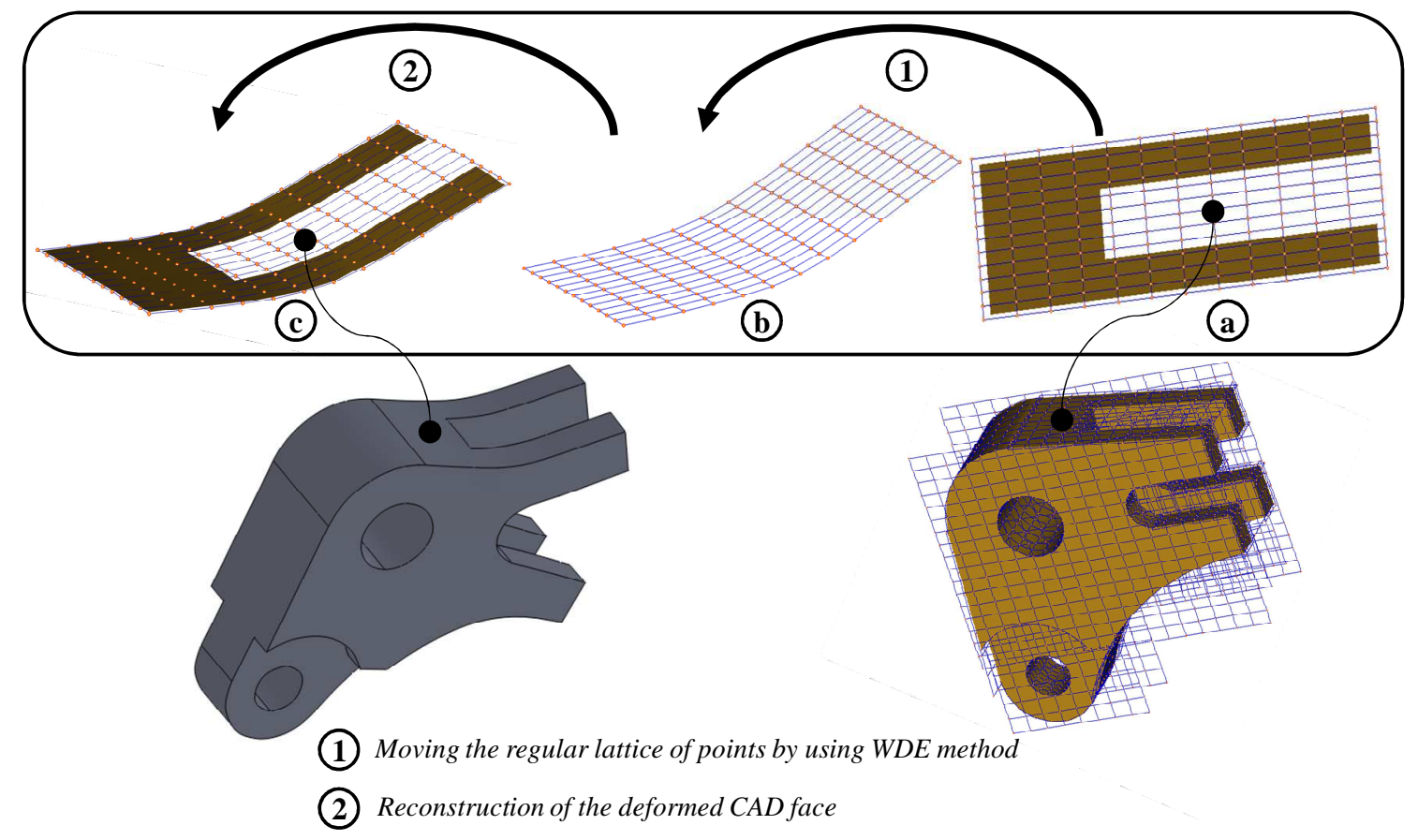

Fig. 12: Face reconstruction is based on three steps - rocker toggle.

Table 2, illustrates the reconstruction error obtained by the reconstruction algorithm of the rocker toggle model (Fig. 11). The reconstruction error is the distance between the interpolation point (node) and its projection on the reconstructed surface. The calculated errors are negligible compared to the node displacements.

Table 2: Quantitative criteria and results (rocker toggle).

\begin{tabular}{|c|c|}
\hline Number of faces & 30 \\
\hline Node displacements (analysis result) & $0-3.0 \mathrm{~mm}$ \\
\hline Maximal reconstruction error & $15.010^{-3} \mathrm{~mm}$ \\
\hline
\end{tabular}




\section{Conclusion}

In this paper, a method of integration between design (CAD) and analysis (FEM) has been introduced. A methodology for updating the CAD model from a FEM analysis result (deformed mesh) has been presented. In the first part, a proposed algorithm of reconstruction is introduced followed by a presentation of the methods used in the reconstruction of CAD entities (faces and edges). The WDE method is used for the reconstruction of the CAD faces.

Using a deformed CAD model, the designer is able to simulate the functioning of the CAD assembly in order to discern possible collisions between parts due to their extreme deformation. The proposed method was evaluated using two case studies: a rod - crankshaft system (assembly) and rocker toggle (part). These examples detail each step, of the proposed method, used to build a CAD model of the deformed part. Our research progresses to improve the WDE method algorithm used for the face reconstruction.

\section{Bibliography}

[1] B. Louhichi, N. Aifaoui, M. Hamdi, A. BenAmara, and V. Francois, An Optimization-based Computational Method for Surface Fitting to Update the Geometric Information of An Existing B-Rep CAD Model, International Journal of CAD/CAM Vol. 9, No. 1, pp. 17-24 (2009).

[2] G. Foucault, J.C. Cuillière, V. Francois, J.C Léon, R. Maranzana, Adaptation of CAD model topology for Finite Element Analysis, Computer Aided Design, 40 (2) (2008) 176-196.

[3] E. Béchet, J.C. Cuillière, F. Trochu, Generation of a Finite Element Mesh from Stereolithography (STL) Files, Computer Aided Design, 34 (2002) 1-17.

[4] Wolfgang A. Wall, Moritz A. Frenzel, Christian Cyron, Isogeometric structural shape optimization, Comput. Methods Appl. Mech. Engrg. 197 (2008) 2976-2988.

[5] Daniel Rypl, Bořek Patzák, Construction of weighted dual graphs of NURBS-based isogeometric meshes, Advances in Engineering Software 60-61 (2013) 31-41.

[6] Bloor M.I.G., Wilson M.J. (1990), Representing PDE surfaces in terms of B-splines, Computer Aided Design 22(6), 324-331.

[7] Qin J., Terzopoulos D. (1996), D-Nurbs: a physics-based framework for geometric design, IEEE Transactions on Visualization and Computer Graphics 2(1), 85-96.

[8] La Areca R., Daniel M. (2004), Declarative approach to Nurbs surface design: from semantic to geometric models, International Conference on Computer Graphics and Artificial Intelligence, Conference proceedings 161-168.

[9] Xinhua Song, Bert Jotter, Modeling and 3D object reconstruction by implicitly defined surfaces with sharp features, Computers \& Graphics 33 (2009) 321-330.

[10] Wei-Cheng Xiea, Xiu-Fen Zoua, Jian-Dong Yangb, Jie-Bin Yangb, Iteration and optimization scheme for the reconstruction of 3D surfaces based on non-uniform rational Bsplines, Computer-Aided Design 44 (2012) 1127-1140.

[11] E.T.Y. Lee, Choosing nodes in parametric curve interpolation, Computer Aided Design, 21

(6) (1989) 363-370.

[12] L. Piegel, W. Tiller, The NURBS BOOK, Springer-Verlag, New York, (1997). 